\title{
Paraspinal Schwannoma of dorsal ramus nerve: A case report
}

\author{
Kunal Ajay Shah*, Ajay Chimanlal Shah \\ Ashutosh Hospital, Vadodara, India
}

A R T I C L E I N F O

\section{Article history:}

Received 19 January 2018

Received in revised form 17 April 2018

Accepted 19 April 2018

Available online 24 April 2018

\section{Keywords:}

Paraspinal schwannoma

Dorsal nerve schwannoma

Intramuscular schwannoma

Extraspinal schwannoma

\begin{abstract}
A B S T R A C T
Spinal Schwannomas are benign nerve sheath tumors and the most common intradural extramedullary tumors of the spine, mainly occurring in the cervical and lumbar region. The location distinct from the spinal canal is very rare. We report a rare case of a 45year old male with Schwannoma in the dorsal paraspinal musculature. Complete surgical excision of the lesion was performed with clear margins and neuropathological analysis revealed a Schwannoma. There has been no recurrence after 6 months.
\end{abstract}

(C) 2018

\section{Introduction}

Schwannomas are homogeneous slow-growing tumors, consisting only of Schwann cells. Schwannomas are mostly benign and less than $1 \%$ become malignant into a form of cancer known as neurofibrosarcoma. ${ }^{1}$ They can also be associated with neurofibromatosis type II. They represent approximately 30\% of all spinal neoplasms and show a male-to female predilection of 1.23 to 1.5:1, slightly favoring males. ${ }^{2-5}$ Intramuscular schwannoma is an extremely rare condition. ${ }^{6}$ Due to low frequency of this tumor type and lack of specific clinical signs and symptoms, pre-surgical diagnosis is difficult. ${ }^{7}$ We present a rare case of intramuscular schwannoma originating from the dorsal ramus nerve in a 45 year old man.

\section{Case report}

A 45 year old male came to the outpatient department with swelling in mid back since the last 10 years (Fig. 1). The swelling was slowly growing over this period. There was no pain associated with the swelling. There was no history of trauma, no sensory symptoms or weakness. There were no constitutional symptoms like low grade fever, loss of weight or anorexia. On examination, there was an $8 \mathrm{~cm} \times 5 \mathrm{~cm}$ sized swelling over right lower dorsal region. There was no tenderness or increased temperature over the swelling. The swelling was firm and had limited mobility. Neurological examination of both lower limbs was normal.

\footnotetext{
* Corresponding author.

E-mail address: kunal@ashutoshhospital.com (K.A. Shah).
}

The MRI showed a well defined encapsulated heterogenous large lesion in the right paraspinal muscles at the level of the D9 through D11 vertebra (Fig. 2). The lesion extended upto the right sided facet joint without intra-foraminal extension. Hypointense foci within the hyperintense area on T2W images, possibly reflecting the fascicular bundles were seen. This is called the Fascicular sign and is positive in Benign nerve sheath tumors.

As the MRI was strongly suggestive of a benign lesion, the patient was planned for excisional biopsy of the lesion. Through a right paramedian incision over the swelling, a large mass was isolated from the surrounding tissue (Fig. 3). The mass was well encapsulated and reached upto the Posterior Spinal elements. The mass was excised and measured $4.6 \times 4.5 \times 7.3 \mathrm{~cm}$ height, length and width respectively (Fig. 4). No originating nerve could be identified.

The gross examination of the tumor showed a smooth outer surface showing a soft, tan, glistening cut surface with focal cystic changes. Microscopic examination revealed a tumor composed of bundles and fascicles of spindle shaped cells with elongated wavy nuclei and nuclear palisading. Verocay bodies were seen (Fig. 5). The stroma showed myxoid areas with cystic change with Antoni A and Antoni B tissue patterns (Figs. 6 and 7).

\section{Discussion}

The differential diagnosis of paraspinal masses includes a variety of lesions like Schwannoma, neurofibroma, meningioma, ependymoma, sarcoma, abscess, tumor arising from a lymphoid, connective and bone tissue. Age, duration, clinical symptoms \& family history are important in narrowing down the differential diagnosis. The most common types of benign soft tissue tumors 


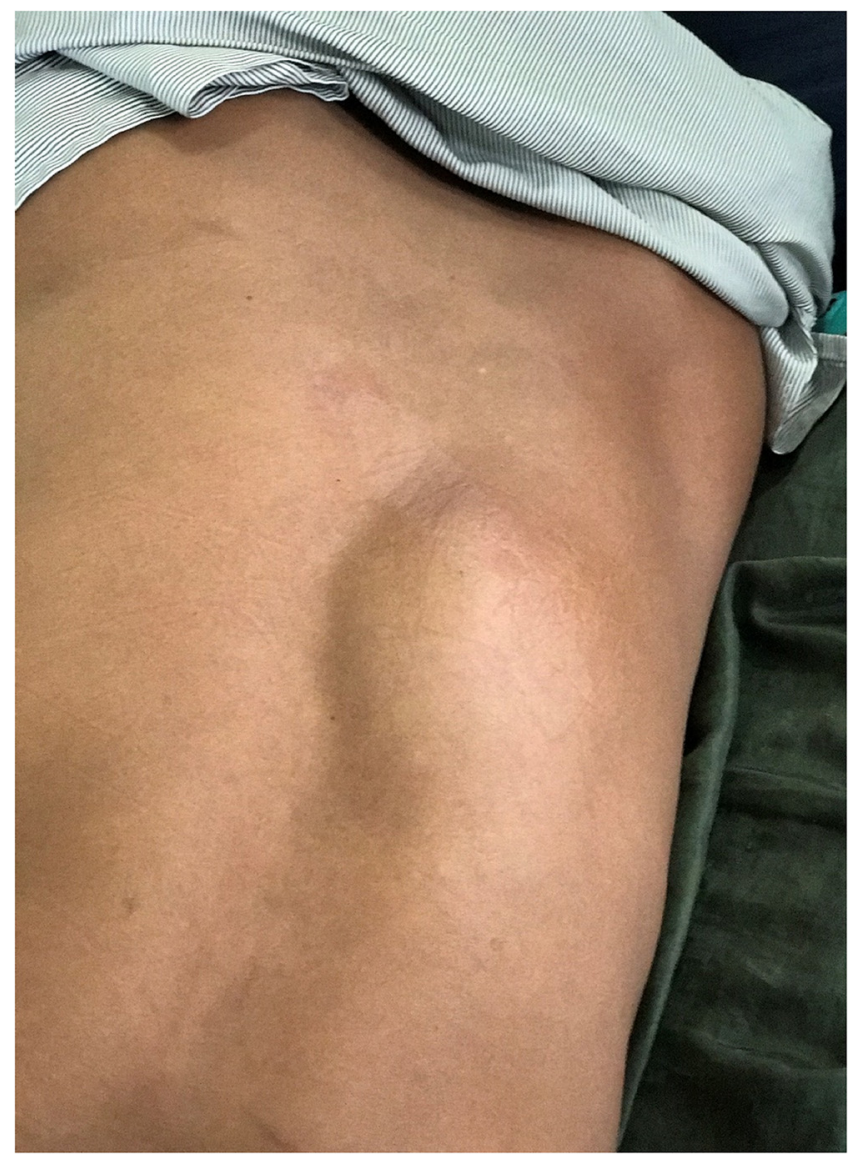

Fig. 1. Swelling over the right paraspinal musculature.

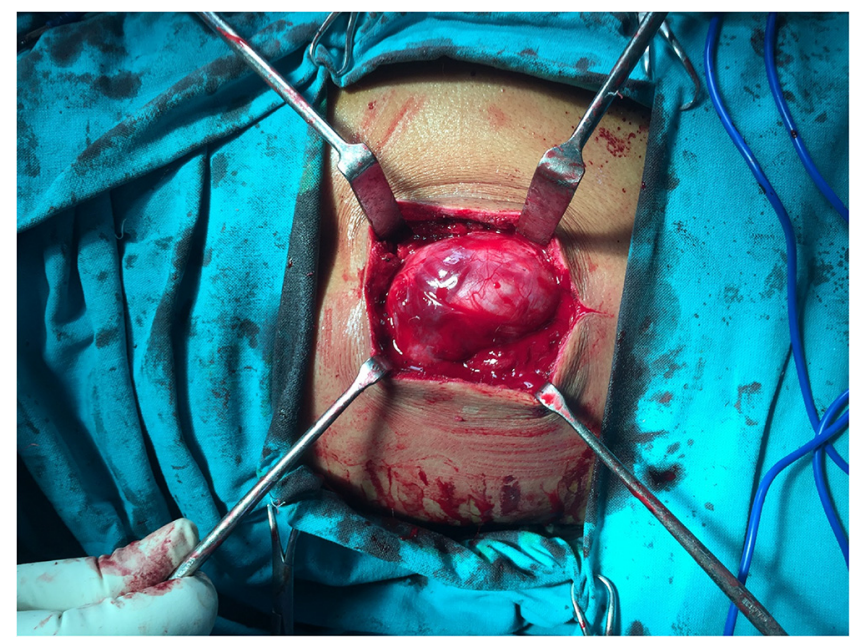

Fig. 3. On dissection, a large, distinct encapsulated mass could be isolated from the surrounding tissue which was extending up to the posterior spinal elements.

are lipoma (16\%), fibrous histiocytoma (13\%), nodular fasciitis (11\%), hemangioma (8\%), and schwannoma (5\%). ${ }^{8}$ Schwannomas may occur at any level of the spinal axis but are mostly located intradurally. Approximately $25-45 \%$ of schwannoma cases occur in the head and neck areas, respectively. ${ }^{9}$ They may extend along the spinal nerve and impose as a dumbbell mass, consisting of an intradural and extradural component. Intramuscular schwannomas are highly uncommon; however, reports have been documented for such tumors originating from the psoas and coracobrachialis muscles, with palpable masses that presented with either no symptoms or lower extremities numbness, but no characteristic symptomatic

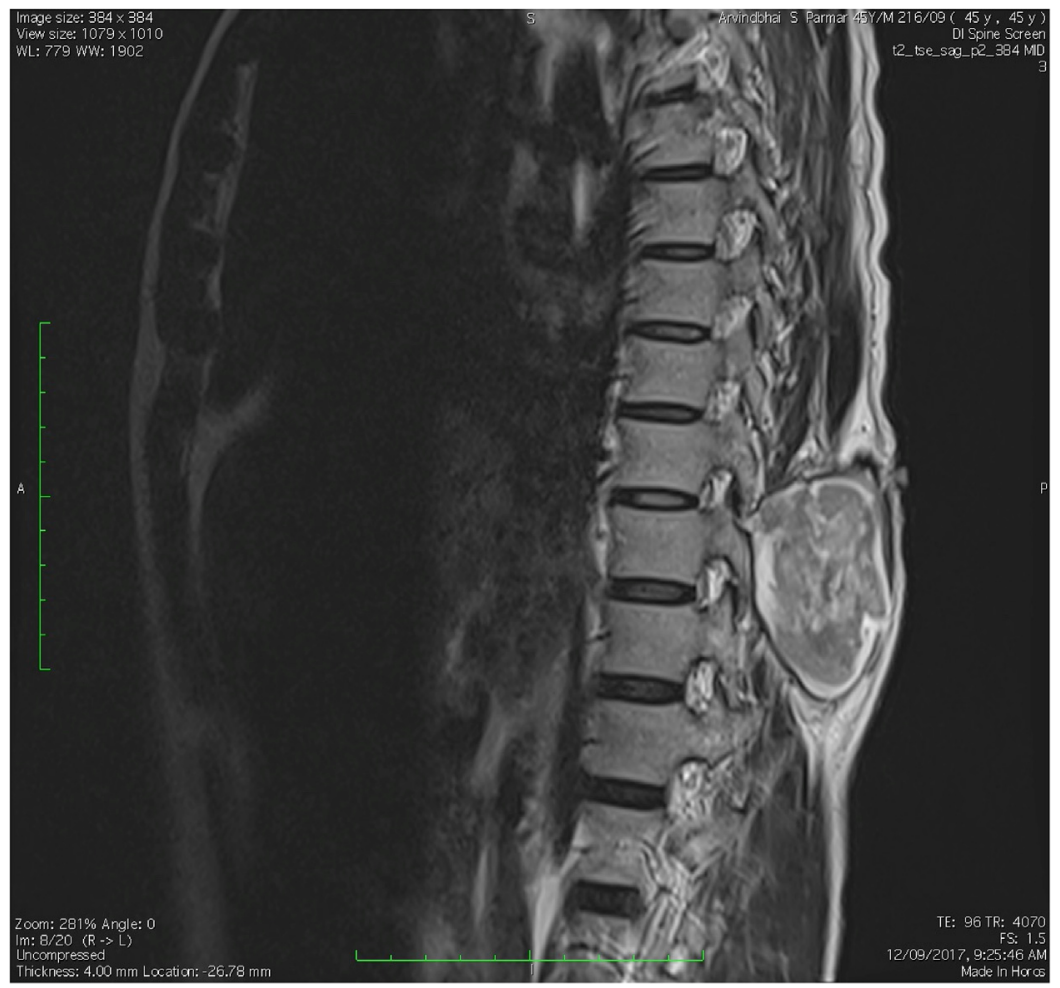

Fig. 2. T2W images Sagittal view shows hypo intense foci within the hyperintense area suggestive of the Fascicular sign. 


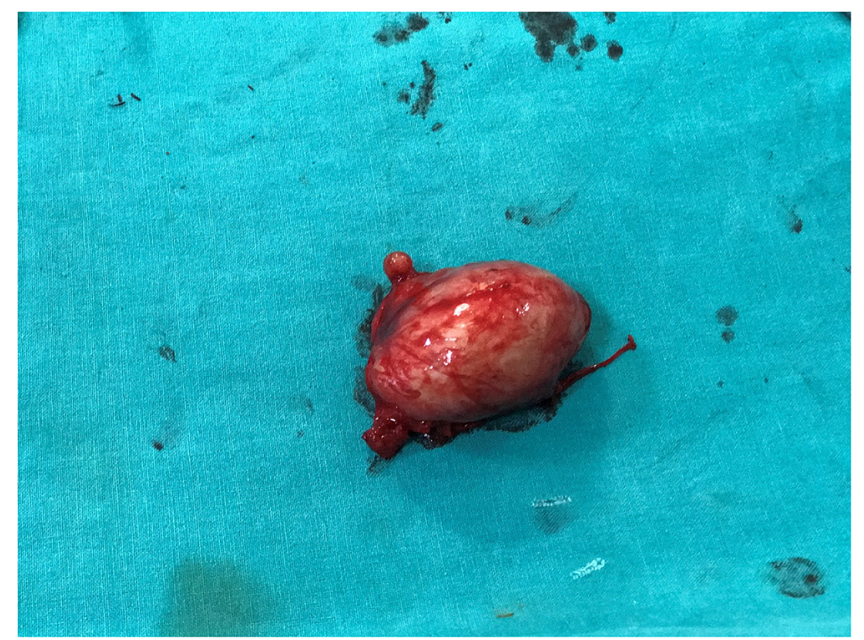

Fig. 4. Excised Mass measured $4.6 \times 4.5 \times 7.3 \mathrm{~cm}$ height, length and width respectively.

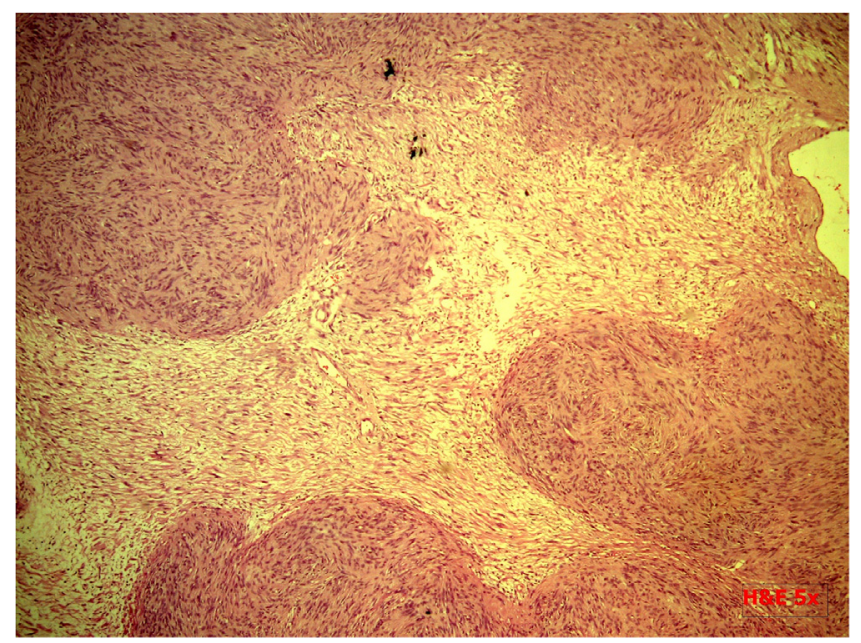

Fig. 5. Classic microscopic appearances of a schwannoma with varying proportions of fascicular and reticular tissues.

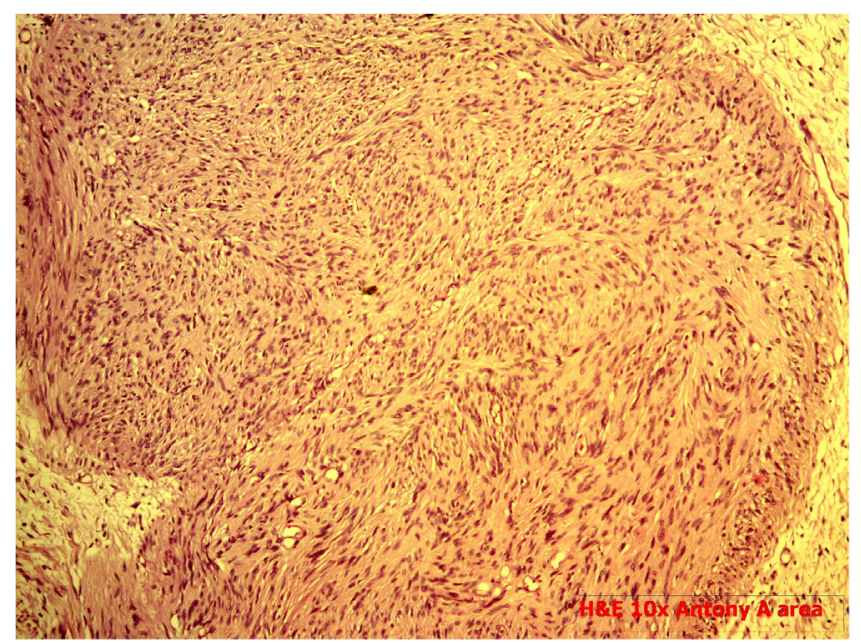

Fig. 6. The more cellular "Antoni A" pattern can be seen.

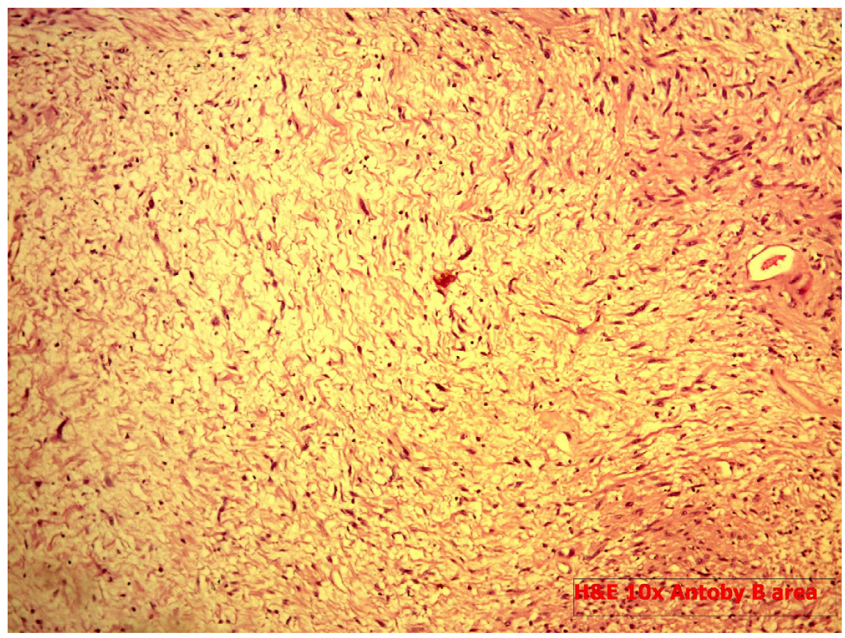

Fig. 7. The “Antoni B" pattern with a looser stroma, fewer cells, and myxoid change.

features. ${ }^{7,10-12}$ However, to the best of our knowledge, only two cases (Table 1) of purely extraspinal Schwannoma of the paraspinal region have been reported previously. ${ }^{13,14}$ Because intramuscular schwannomas originate from motor branch nerves, neurological symptoms, including pain, motor weakness, paresthesia, are rare. ${ }^{6,15}$ In our case, there was no radicular pain, motor weakness; or tenderness. In our patient, intramuscular schwannoma occurred in the erector spinae muscles. The erector spinae muscles are dominated by the dorsal ramus nerve, which branches to form the spinae nerve. Schwannomas are typically well encapsulated and solid with membranes composed of a thin fibrous capsule. In our case, we observed a typical schwannoma pattern, with a relatively large tumor. Although no originating nerve could be identified, it was most likely arising from the dorsal ramus nerve.

Distribution of the lesion along the course of the nerve is a feature of prime importance for the diagnosis of a nerve sheath tumor. Schwannomas are located eccentrically in relation to the nerve, in contradiction to neurofibroma, which is central and inseparable from the parent nerve. ${ }^{16}$ The 'fascicular sign' for the neurogenic lesions is represented by multiple ring-like structures, which appear as hypo intense foci within the hyperintense area on T2W images, possibly reflecting the fascicular bundles seen histologically. This sign is often seen in benign nerve sheath tumors. ${ }^{16}$ Our case showed the Fascicular sign and the tumor was eccentrically located.

The histological hallmark of a schwannoma is varying proportions of two distinctive tissues: fascicular type (Antoni-A) and reticular type (Antoni-B). ${ }^{6,17}$ Antoni $A$ and $B$ tissue types represent distinct histologic architectural patterns that aid in the histopathologic diagnosis of schwannomas. Type A tissue is highly cellular and demonstrates nuclear palisading and associated Verocay bodies, which reflects their prominent extracellular matrix and secretion of laminin. The increased basement membrane structures in Antoni A areas are rich in laminin, a high molecular weight glycoprotein which is produced by Schwann cells and therefore seen in Schwannomas and to lesser extent in neurofibromas. ${ }^{18}$ Immunohistochemical demonstration of laminin in the tumor serves to reliably differentiate Schwann cell derived lesions like Schwannomas from other histologically similar looking lesions like histiocytomas and leiomyomas and their malignant counterparts fibrosarcomas and leiomyosarcomas. Antoni B tissue on the other hand is less cellular, with a myxomatous stroma in which are scattered loosely arranged cells. Cells within the Antoni $\mathrm{B}$ regions are often thin and wispy and are separated from other 
Table 1

Review of literature on paraspinal schwannoma in musculature.

\begin{tabular}{|c|c|c|c|c|c|}
\hline Study & Age & Sex & Site & Surgery & Comments \\
\hline Victoria Ohla et al. ${ }^{13}$ & 34 & Female & Lumbar Spine & Complete Excision \& Biopsy confirmation & No recurrence after 6 months \\
\hline Jeong Hoon Kim et al. ${ }^{14}$ & 62 & Female & Dorsal Spine & Biopsy confirmation and then Complete Excision & No mentioned follow up \\
\hline Current Study (2017) & 45 & Male & Dorsal Spine & Complete Excision and Biopsy confirmation & No recurrence at 6 months \\
\hline
\end{tabular}

cells by microcystic spaces filled with basophilic mucin. A typical Verocay body consists of a stacked arrangement of two rows of elongated palisading nuclei that alternates with acellular zones made up of cytoplasmic processes of the Schwann cells. The pathogenesis of the formation of this structure is explained by the overexpression of laminins in the cells that make up the Verocay body. ${ }^{18}$ Immunohistochemically, schwannomas typically show diffuse, strongly S-100 protein expression. ${ }^{17}$

In the present case, as the MRI findings were indicative of a benign nerve sheath tumour, we cautiously removed the encapsulated mass from the origin but could not identify the dorsal ramus nerve. Our tissue biopsy confirmed the presence of a very rare intramuscular benign schwannoma, with typical features.

\section{Conclusion}

When a patient has palpable, deep or intrinsic back muscle tissue mass, a radiological examination, such as a computed tomography (CT) or MRI should be considered. In an MRI, a distinct pattern in the surrounding wall of the mass is suggestive of schwannoma. Although intradural-extramedullary Schwannomas are frequently encountered in clinical practice, we should also be aware of a purely paraspinal location. To our knowledge, this is only the second report of a dorsal ramus nerve schwannoma within the erector spinae muscles. If histological findings suggest the presence of Antoni-A or Antoni-B and the S-100 protein is strongly expressed, a diagnosis of dorsal ramus schwannoma is confirmed. As seen in our case complete surgical excision can be considered curative and should hence be considered as the treatment of choice.

\section{Conflict of interest and financial disclosures}

None.

\section{References}

1. Bhatia M, Nirhale DS, et al. Schwannoma over submandibular region: a rare presentation. Trop J Med Res. 2015;18:48-50.

2. Riffaud L, Morandi X, Massengo S, et al. MRI of intramedullary spinal schwannomas: case report and review of the literature. Neuroradiology. 2000:42:275-279.

3. George B, Lot G. Neurinomas of the first two cervical nerve roots: a series of 42 cases. J Neurosurg. 1995;82:917-923.

4. Lot G, George B. Cervical neuromas with extradural components: surgical management in a series of 57 patients. Neuro Surg. 1997;41:813-820 discussion 820-822.

5. Antinheimo J, Sankila R, Carpen O, et al. Population based analysis of sporadic and type 2 neurofibromatosis-associated meningiomas and schwannomas. Neurology. 2000;54:71-76.

6. Nishio J, Ueki T, Naito M. Intramuscular schwannoma of the musculocutaneous nerve: an uncommon clinical presentation. Exp Ther Med. 2013;6:164-166.

7. D'Silva KJ, Dwivedi AJ, Barnwell JM. Schwannoma of the psoas major muscle presenting with abdominal and back pain. Dig Dis Sci. 2003;48:1619-1621.

8. Kransdorf MJ. Benign soft-tissue tumors in a large referral population: distribution of specific diagnoses by age, sex, and location. Am J Roentgenol. 1995;164:395-402.

9. Sahoo PK, Mandal PK, Ghosh S. Schwannoma of the hard palate. Natl J Maxillofac Surg. 2014;5:39-41.

10. Hsu YC, Shih YY, Gao HW, et al. Intramuscular schwannoma arising from the psoas muscle presenting with femoral nerve neuropathy. South Med J. 2010;103:477-479.

11. Knight DM, Birch R, Pringle J. Benign solitary schwannomas: a review of 234 cases. J Bone Jt Surg Br. 2007;89:382-387.

12. Muramatsu K, Ihara K, Yoshida Y, et al. Intramuscular schwannoma arising from the psoas major muscle. Clin Neurol Neurosurg. 2008;110:532-533.

13. Ohla V, Scheiwe C. A purely paraspinally located schwannoma from a dorsal root of a spinal nerve. Br J Med Med Res. 2014;4(6):1366-1370.

14. Kim Jeong Hoon, Cho Tack Geun, et al. Erector spinal muscular schwannoma of the dorsal ramus nerve: a case report. Korean J Spine. 2015;12(3):204-206.

15. Kwon BC, Baek GH, Chung MS, et al. Intramuscular neurilemoma. J Bone Jt Surg Br. 2003;85:723-725.

16. Koteshwara Prakashini, Bajpai Surabhi, Kakkar Chandan, et al. Telltale signs of peripheral neurogenic tumors on magnetic resonance imaging. Indian J Radiol Imaging. 2015;25(October-December (4)):453-458.

17. Rodriguez FJ, Folpe AL, et al. Pathology of peripheral nerve sheath tumors: diagnostic overview and update on selected diagnostic problems. Acta Neuropathol. 2012;123:295-319.

18. Joshi R. Learning from eponyms: Jose Verocay and Verocay bodies, Antoni A and B areas, Nils Antoni and Schwannomas. Indian Dermatol Online J. 2012;3:215-219. 